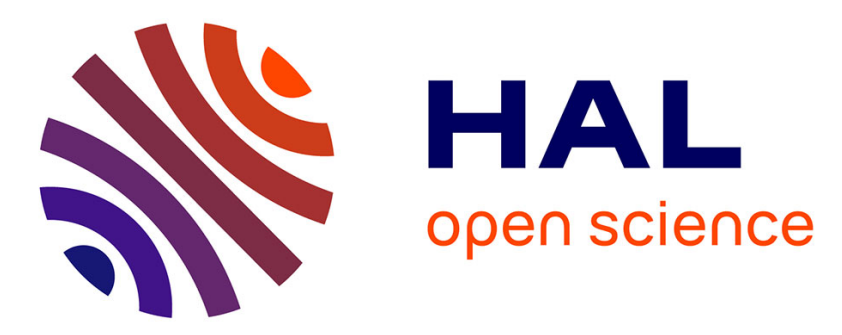

\title{
Optimization of the second order autoregressive model AR(2) for Rayleigh-Jakes flat fading channel estimation with Kalman filter
}

Ali Houssam El Husseini, Eric Pierre Simon, Laurent Ros

\section{- To cite this version:}

Ali Houssam El Husseini, Eric Pierre Simon, Laurent Ros. Optimization of the second order autoregressive model AR(2) for Rayleigh-Jakes flat fading channel estimation with Kalman filter. DSP 2017 - 22th IEEE International Conference on Digital Signal Processing, IEEE, Aug 2017, Londres, United Kingdom. hal-01576842

\section{HAL Id: hal-01576842 \\ https://hal.science/hal-01576842}

Submitted on 24 Aug 2017

HAL is a multi-disciplinary open access archive for the deposit and dissemination of scientific research documents, whether they are published or not. The documents may come from teaching and research institutions in France or abroad, or from public or private research centers.
L'archive ouverte pluridisciplinaire HAL, est destinée au dépôt et à la diffusion de documents scientifiques de niveau recherche, publiés ou non, émanant des établissements d'enseignement et de recherche français ou étrangers, des laboratoires publics ou privés. 


\section{Optimization of the second order autoregressive model AR(2) for Rayleigh-Jakes flat fading channel estimation with Kalman filter.}

\author{
Ali Houssam EL HUSSEINI \\ IEMN lab, Group Telice \\ University of Lille 1 \\ 59650, Villeneuve d'Ascq, France \\ Email: ali.housseini@outlook.com
}

\author{
Eric Pierre SIMON \\ IEMN lab, Group Telice \\ University of Lille 1 \\ 59650, Villeneuve d'Ascq, France \\ Email: eric.simon@univ-lille1.fr
}

\author{
Laurent ROS \\ GIPSA-lab \\ Image and signal Department, \\ BP46, 38402 Saint-Martin d'Hres, France \\ Email : laurent.ros@gipsa-lab.grenoble-inp.fr
}

\begin{abstract}
This paper deals with the estimation of the flat fading Rayleigh channel with Jakes' Doppler spectrum (model due to R.H. Clarke in 1968) and slow fading variations. A common method in literature consists in approximating the variations of the channel using an auto-regressive model of order p $(\operatorname{AR}(p))$, whose parameters are adjusted according to the "correlation matching" (CM) criterion and then estimated by a Kalman filter (KF). Recent studies based on first order AR(1) showed that the performance is far from the Bayesian CramerRao bound for slow to moderate channel variations, which is the case for many applications. The same studies on first order model have shown the importance of replacing the $\mathrm{CM}$ criterion with a MAV criterion (Minimization of Asymptotic Variance). Moreover, it has been shown in literature that increasing the order of the model by passing from $\operatorname{AR}(1)$ to $\operatorname{AR}(2)$ did not improve the performance when the $\mathrm{CM}$ criterion is considered. In order to obtain an improvement in performance, it is essential to consider the MAV criterion with second order autoregressive model $\mathbf{A R}(2)$, as shown in this article. By imposing a linear relation between one of the parameters and the Doppler frequency, we derive analytic formulas for suboptimal adjustment of the parameters of $\operatorname{AR}(2)$ as a function of the noise level and the Doppler frequency of the channel. Theoretical assumptions are validated via simulation.

Index Terms-Rayleigh channel estimation, Autoregressive model, Jakes Doppler spectrum, Flat fading, Kalman filter, steady-state.
\end{abstract}

\section{INTRODUCTION}

This paper deals with channel estimation for radio-mobile communications. The problem is the estimation of a channel which evolves according to a Rayleigh random model with Jakes Doppler spectrum (most common model due to R.H. Clarke in 1968 [1]). Conventionally, the channel estimation is performed with Kalman filter (KF), which requires choosing state model to approximate channel variations. The most commonly state model used in the literature is autoregressive model of order $\mathrm{p}(\mathrm{AR}(\mathrm{p}))$, whose parameters are adjusted according to correlation matching criterion (CM) [2] ${ }^{1}$. However, the performance in terms of distance with the Bayesian

\footnotetext{
${ }^{1}$ Note also that this method is used to approximate the correlated fading channels is also used today for the computer simulation of the "Rayleigh fading channel model with Jakes' Doppler spectrum", as for example in one version of the function "Rayleighchan" in Matlab.
}

Cramer-Rao Bound (BCRB) is disappointing in the case of a slow to moderate channel variation. It has been shown in [3], [4] that even by increasing the order of the AR model from $p=1$ to $p=2$ or 3 , the performance of the estimation stagnates (see [3] -Fig B.2 and [4] -Fig 3.5 ). The authors of [2] proposed to correct the $\mathrm{CM}$ criterion by adding a very small parameter $\epsilon$ on the diagonal of the correlation matrix to improve its conditioning, which makes it possible to improve the performance considerably. However, the $\epsilon$ parameter is set only by simulation and the mean square error (MSE) curve is still far from the BCRB. Recent work has focused on another state model, the random walk (RW) model with the minimization of the asymptotic variance (MAV) criterion [5]-[7]. These studies showed that switching from order 1 to a higher order allowed to obtain a significant gain in performance. In addition, the KF with RW set by MAV had a lower mean squared error (MSE) than the KF with AR set by CM. Moreover, these studies had the advantage of providing the analytical formulas for the optimal adjustment of the model parameters. Although the RW and AR models have notable differences (the RW model is nonstationary in contrast to the AR model, and is mainly referred to model the phase evolution which is modulo $2 \pi$ ), we can assume that the use of a well tuned second order autoregressive model $\mathrm{AR}(2)$ must also allow a significant improvement over the first order autoregressive model AR(1). However, eventhough the AR model is the most used in literature to model and estimate the channel, there is no analytical work on the optimal setting (according to MAV criterion instead of CM) of the second order autoregressive model in this context. Such work has recently been carried out with promising results, but only with the AR model of first order AR(1) [3]. We propose to extend this work to the second order model. Thus, analytical formulas for setting parameters are established, and it is shown that the passage from a first order to a well tuned second order effectively allows a marked increase in the performance of the estimation in terms of MSE.

This paper is organized as follows. Section 2, describes the system model. In section 3, the Kalman filter and the steady 
state are discussed. Section 4 and 5 present respectively the results and conclusion of this paper.

\section{SySTEM MODEL}

We consider the estimation of a flat Rayleigh fading channel. The observation is ${ }^{2}$ :

$$
y_{(k)}=\alpha_{(k)}+w_{(k)}
$$

where $k$ is the time index, $w_{(k)}$ is a zero-mean additive white circular complex Gaussian noise with variance $\sigma_{w}^{2}$ and $\alpha_{(k)}$ is a zero-mean correlated circular complex Gaussian channel gain with variance $\sigma_{\alpha}^{2}$. The signal to noise ratio is $\mathrm{SNR}=$ $\sigma_{\alpha}^{2} / \sigma_{w}^{2}$ and the normalized Doppler frequency of this channel is $f_{d} T$, where $T$ is the symbol period. The Jakes' Doppler spectrum for this channel is:

$$
\Gamma(f)= \begin{cases}\frac{\sigma_{\alpha}^{2}}{\pi f_{d} \sqrt{1-\left(\frac{f}{f_{d}}\right)^{2}}} & \text { if }|f|<f_{d} . \\ 0 & \text { if }|f|>f_{d} .\end{cases}
$$

The autocorrelation coefficient $R_{\alpha}[m]$ of the stationnary channel gain $\alpha$ is defined for a lag $\mathrm{m}$ by :

$$
R_{\alpha}[m]=E\left\{\alpha_{(k)} \cdot \alpha_{(k-m)}^{*}\right\}=\sigma_{\alpha}^{2} J_{0}\left(2 \pi f_{d} T m\right)
$$

where $J_{0}$ is the zeroth-order Bessel function of the first Kind. In this article we approximate the channel gain $\alpha_{(k)}$ by a second order autoregressive model AR(2):

$$
\tilde{\alpha}_{(k)}=a_{1} \tilde{\alpha}_{(k-1)}+a_{2} \tilde{\alpha}_{(k-2)}+u_{(k)}
$$

where $\tilde{\alpha}_{(k)}$ is the approximation of $\alpha_{(k)} \operatorname{AR}(2)$ and $u_{(k)}$ is a white circular complex Gaussian state noise with variance $\sigma_{u}^{2}=R_{\tilde{\alpha}}[0]-a_{1} R_{\tilde{\alpha}}[1]-a_{2} R_{\tilde{\alpha}}[2]$. This expression is calculated using Yule-Walker equations [8]. It is assumed that $R_{\tilde{\alpha}}[0]=$ $\sigma_{\alpha}^{2}$. These equations also give $R_{\tilde{\alpha}}[1]=\frac{a_{1} R_{\tilde{\alpha}}[0]}{1-a_{2}}$ and $R_{\tilde{\alpha}}[2]=$ $a_{1} R_{\tilde{\alpha}}[1]+a_{2} R_{\tilde{\alpha}}[0]$.

Using these expressions we obtain $\sigma_{u}^{2}$ only as a function of $a_{1}$ and $a_{2}$, which will be useful for the following:

$$
\sigma_{u}^{2}=\sigma_{\alpha}^{2} \frac{\left(1+a_{2}\right)\left(1-a_{1}-a_{2}\right)\left(1+a_{1}-a_{2}\right)}{\left(1-a_{2}\right)}
$$

By passing to the $z$-transformation of the equation (4), the transfer function of the $\mathrm{AR}(2)$ model is:

$$
H(z)=\frac{1}{1-a_{1} z^{-1}-a_{2} z^{-2}}
$$

In our case Jakes model is approximated by the $\operatorname{AR}(2)$ model.In order to design low-pass filter with a peak around $f_{d} T$, we need to place a set of complex conjugate poles in the

\footnotetext{
${ }^{2}$ Model (1) assumes that symbols are normalized and known (or decided), additionally to flat fading assumption. Although this model is admittedly simplistic, it can be applied to different (more involved contexts), such as pilot-aided multicarrier systems in frequency-selective wireless channels [6].
}

$z$-plane at $z_{1}=r \cdot \mathrm{e}^{-j 2 \pi f_{\mathrm{AR}(2)} T}$ and $z_{2}=r \cdot \mathrm{e}^{+j 2 \pi f_{\mathrm{AR}(2)} T}[9]$, [10]:

$$
\begin{aligned}
H(z) & =\frac{1}{\left(1-z_{1} z^{-1}\right)\left(1-z_{2} z^{-1}\right)} \\
& =\frac{1}{1-2 r \cos \left(2 \pi f_{\mathrm{AR}(2)} T\right) z^{-1}+r^{2} z^{-2}}
\end{aligned}
$$

Comparing equations (6) and (7) we have :

$$
a_{1}=2 r \cos \left(2 \pi f_{\mathrm{AR}(2)} T\right) \quad a_{2}=-r^{2}
$$

With $f_{\text {AR (2) }} T$ close to $f_{d} T \ll 1$ and $r$ close to 1 , we obtain $a_{1}$ close to 2 and $a_{2}$ close to -1 [9], with $\left|a_{1}\right|<2$ and $\left|a_{2}\right|<1$ to ensure stationarity [11]. These orders of magnitude for $a_{1}$ and $a_{2}$ will be used in our theoretical developments to obtain simplified analytical formulas. [9] chooses $f_{\mathrm{AR}(2)} T=f_{d} T$, while [10] chooses $f_{\mathrm{AR}(2)} T=\frac{1}{\sqrt{2}} f_{d} T$. However, in both cases no expression is given for the choice of $r$.

In the following, we consider channel estimation with KF. The criterion for tuning the model parameters is based on an approximation of the minimization of the mean square error (MSE) in steady state mode, $\sigma_{\epsilon}^{2}=E\left\{\left|\alpha_{(k)}-\hat{\alpha}_{(k \mid k)}\right|^{2}\right\}$, where $\hat{\alpha}_{(k \mid k)}$ is the estimate of $\alpha_{(k)}$ and " $k \rightarrow+\infty$ ".

\section{KALMAN FILTER}

\section{A. Kalman filter equations}

The second order autoregressive model can be reformulated in a state model. The state vector to be considered includes the channel gain at $k$ and $k-1, \boldsymbol{\alpha}_{(k)}=\left[\alpha_{(k)}, \alpha_{(k-1)}\right]^{T}$ and $\tilde{\boldsymbol{\alpha}}_{(k)}=\left[\tilde{\alpha}_{(k)}, \tilde{\alpha}_{(k-1)}\right]^{T}$. The state transition matrix is $\mathbf{M}=$ $\left[\begin{array}{cc}a_{1} & a_{2} \\ 1 & 0\end{array}\right]$ and the state noise vector is : $\mathbf{u}_{(k)}=\left[u_{(k)}, 0\right]^{T}$. The selection vector of size $1 \times 2$ is $\mathbf{s}^{T}=[1,0]$. The state evolution of (4) and observation (1) becomes:

$$
\begin{gathered}
\tilde{\boldsymbol{\alpha}}_{(k)}=\mathbf{M} \tilde{\boldsymbol{\alpha}}_{(k-1)}+\mathbf{u}_{(k)} \\
y_{(k)}=\mathbf{s}^{T} \boldsymbol{\alpha}_{(k)}+w_{(k)}
\end{gathered}
$$

Regarding the state-space formulation (9) and (10), the two stages of the filter are :

\section{Time update equations:}

$$
\begin{gathered}
\hat{\boldsymbol{\alpha}}_{(k \mid k-1)}=\mathbf{M} \hat{\boldsymbol{\alpha}}_{(k-1 \mid k-1)} \\
\mathbf{P}_{(k \mid k-1)}=\mathbf{M} \mathbf{P}_{(k-1 \mid k-1)} \mathbf{M}^{H}+\mathbf{U}
\end{gathered}
$$

\section{Measurement update equations:}

$$
\begin{gathered}
\mathbf{K}_{(k)}=\frac{\mathbf{P}_{(k \mid k-1)} \mathbf{s}}{\mathbf{s}^{T} \mathbf{P}_{(k \mid k-1)} \mathbf{s}+\sigma_{w}^{2}} \\
\hat{\boldsymbol{\alpha}}_{(k \mid k)}=\hat{\boldsymbol{\alpha}}_{(k \mid k-1)}+\mathbf{K}_{(k)}\left(y_{(k)}-\mathbf{s}^{T} \hat{\boldsymbol{\alpha}}_{(k \mid k-1)}\right) \\
\mathbf{P}_{(k \mid k)}=\left(\mathbf{I}_{2}-\mathbf{K}_{(k)} \mathbf{s}^{T}\right) \mathbf{P}_{(k \mid k-1)}
\end{gathered}
$$

where $\mathbf{K}_{(k)}=\left[\begin{array}{l}K_{1(k)} \\ K_{2(k)}\end{array}\right]$ is the Kalman gain vector, $\mathbf{U}=$ $\left[\begin{array}{cc}\sigma_{u}^{2} & 0 \\ 0 & 0\end{array}\right], \mathbf{I}_{2} 2 \times 2$ identity matrix and $\mathbf{P}_{(k \mid k)}$ and $\mathbf{P}_{(k \mid k-1)}$ 
are respectively the $2 \times 2$ a posteriori and predicted error covariance matrices.

\section{B. Kalman filter steady-state}

Since the linear system is observable and controlable, an asymptotic regim is quickly reached for which the covariances and gain of the filter become constant :

$\mathbf{K}_{(k)}=\mathbf{K}_{(k+1)}=\mathbf{K}_{\infty}=\left[\begin{array}{c}K_{1} \\ K_{2}\end{array}\right], \mathbf{P}_{(k \mid k)}=\mathbf{P}_{(k+1 \mid k+1)}=$ $\mathbf{P}_{\infty}=\left[\begin{array}{ll}P_{11} & P_{12} \\ P_{21} & P_{22}\end{array}\right]$ and $\mathbf{P}_{(k \mid k-1)}=\mathbf{P}_{(k+1 \mid k)}=\mathbf{P}_{\infty}^{\prime}=$ $\left[\begin{array}{ll}P_{11}^{\prime} & P_{12}^{\prime} \\ P_{21}^{\prime} & P_{22}^{\prime}\end{array}\right]$

The objective of this section is to compute $K_{1}$ as a function of $\sigma_{u}^{2}$ (itself a function of $a_{1}$ and $a_{2}$ ) to be able to do the optimization in the following section. For this reason, it is necessary to solve the Ricatti's equations (12) (13) (15) in $P_{11}^{\prime}$ by manipulating the previous equations. A quartic equation is obtained after calculation :

$$
-P_{11}^{\prime 4}+A P_{11}^{\prime 3}+B P_{11}^{\prime 2}+C P_{11}^{\prime}+D=0
$$

where A, B, C and D are coefficients in terms of $\sigma_{w}^{2}, \sigma_{u}^{2}$, $a_{1}$ and $a_{2}$. This equation is hard to solve or to approximate due to the large numbers of coefficients and the complexity of the method used to solve this equation [12]. In [13], it is demonstrated that the solution of equations (12) and (15) is given by the eigen vector of the following matrice :

$$
\mathbf{H}_{\mathbf{f}}=\left[\begin{array}{cc}
\boldsymbol{\Phi}^{-T} & \boldsymbol{\Phi}^{-T} \mathbf{R}_{\mathbf{f}}^{\dagger} \\
\mathbf{Q}_{\mathbf{f}}{ }^{*} \boldsymbol{\Phi}^{-T} & \boldsymbol{\Phi}+\mathbf{Q}_{\mathbf{f}}^{*} \boldsymbol{\Phi}^{-T} \mathbf{R}_{\mathbf{f}}^{\dagger}
\end{array}\right]
$$

where $\boldsymbol{\Phi}^{-T}=\mathbf{M}^{-T}, \mathbf{R}_{\mathbf{f}}^{\dagger}=\frac{\mathbf{s} \cdot \mathbf{s}^{T}}{\sigma_{w}^{2}}$ and $\mathbf{Q}_{\mathbf{f}}^{*}=\frac{\mathbf{s}^{T} \cdot \mathbf{s}}{\sigma_{u}^{2}}$.

[14] gives an analytical solution based on the work of [13]. In our case and by applying the same methods used in [13], [14] we get the approximateed expression:

$$
K_{1} \simeq \sqrt{\frac{2 \sigma_{u}}{\sigma_{w}}}
$$

where we have used the following assumptions for the approximation: $\frac{\sigma_{u}^{4}}{\sigma_{w}^{4}} \ll \frac{\sigma_{u}^{2}}{\sigma_{w}^{2}} \ll \frac{\sigma_{u}}{\sigma_{w}} \ll \sqrt{\frac{\sigma_{u}}{\sigma_{w}}} \ll 1, a_{1} \backsim 2$ and $a_{2} \simeq-1$.

This approximation is validated by simulation in figure (1). In this figure we see that the approximation (18) is close to the asymptotic value of $K_{1}$ obtained by Monte-Carlo simulation in all the range of usual SNR.

\section{Variance of MSE in steady-state and optimization}

$L(z)$ is the transfer function of the filter which gives $\hat{\alpha}_{(k \mid k)}$ with the observation $y_{(k)}$ as input. In a steady state, the solution is given by the system of equations (11) and (14) of the KF by:

$$
L(z)=\frac{K_{1}+a_{2} K_{2} z^{-1}}{1+z^{-1}\left(a_{2} K_{2}-a_{1}\left(1-K_{1}\right)\right)-a_{2}\left(1-K_{1}\right) z^{-2}}
$$

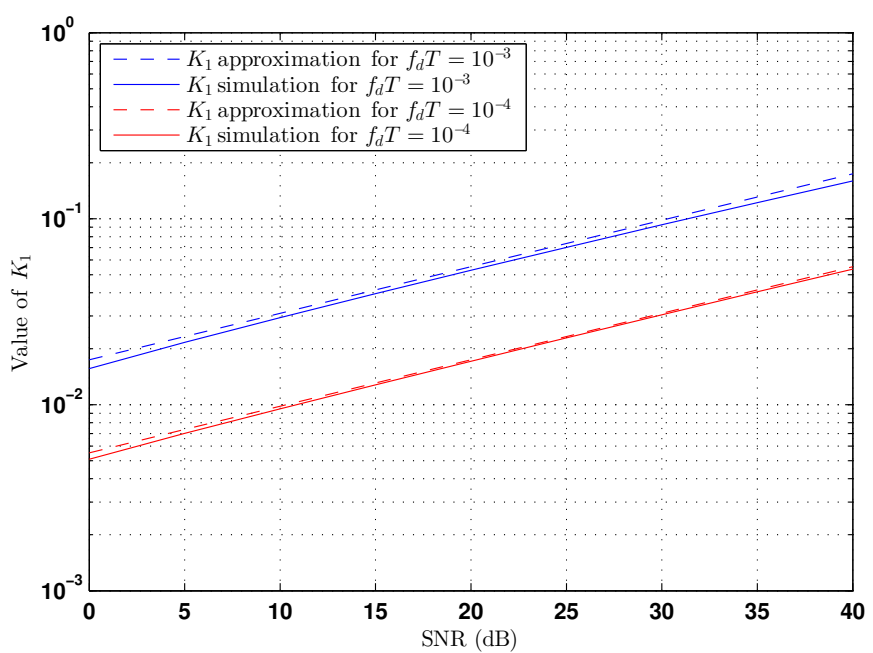

Fig. 1: Comparison between $K_{1}$ (simulation) and $K_{1}$ (approximation (18)) for $f_{d} T=10^{-4}$ and $f_{d} T=10^{-3}$ in terms of $\operatorname{SNR}\left(\sigma_{u}\right.$ is tuned according to eq. (25)).

where $K_{2}=\frac{a_{1}\left(1-K_{1}\right) K_{1}}{1-a_{2}+a_{2} K_{1}}$.

From the filtering equations, we have $\hat{\alpha}(z)=L(z)(\alpha(z)+$ $w(z))$ and the estimation error :

$$
\epsilon(z)=\alpha(z)-\hat{\alpha}(z)=(1-L(z)) \alpha(z)-L(z) w(z)
$$

Therefore, it remains to calculate the power of the error from $\epsilon(z)(20)$ :

$$
\sigma_{\epsilon}^{2}=\sigma_{\epsilon \alpha}^{2}+\sigma_{\epsilon w}^{2}
$$

- the dynamic error variance $\sigma_{\epsilon \alpha}^{2}$ is due to the variations of $\alpha_{(k)}$ filtered by the high pass filter $1-L(z)$ :

$$
\begin{aligned}
\sigma_{\epsilon \alpha}^{2} & \stackrel{\text { def }}{=} \int_{-f_{d}}^{+f_{d}}\left|1-L\left(e^{2 i \pi f T}\right)\right|^{2} \Gamma_{\alpha}(f) d f \\
& \simeq \int_{-f_{d}}^{+f_{d}} \frac{(2 \pi f T)^{4}}{K_{1}^{4}} \Gamma_{\alpha}(f) d f=\sigma_{\alpha}^{2} \frac{6 \pi^{4}\left(f_{d} T\right)^{4}}{K_{1}^{4}}
\end{aligned}
$$

The approximation in (22) is made by using the previous approximation of $K_{1}$, and in assuming low Doppler normalized frequency $f_{d} T \ll 1$ and $K_{1}^{4} \ll K_{1}^{3} \ll K_{1}^{2} \ll K_{1} \ll 1$, $f_{d} T^{4} \ll f_{d} T^{3} \ll f_{d} T^{2} \ll f_{d} T \ll 1, a_{1} \simeq 2, a_{2} \simeq-1$ and $z=e^{2 i \pi f T} \simeq 1+i 2 \pi f T$. The exact integration is then computed from $\Gamma_{\alpha}(f)$ defined in (2) by changing variable : $\cos (\theta)=\left(f / f_{d}\right)$.

- the static error variance $\sigma_{\epsilon w}^{2}$ is due to additive noise $w_{(k)}$ filtered by the low pass filter $L(z)$ :

$$
\begin{aligned}
\sigma_{\epsilon w}^{2} & \stackrel{\text { def }}{=} \sigma_{w}^{2} T \int_{-\frac{1}{2 T}}^{\frac{1}{2 T}}\left|L\left(e^{2 i \pi f T}\right)\right|^{2} d f \\
& \simeq \sigma_{w}^{2} \frac{3 K_{1}}{4}
\end{aligned}
$$


The exact integration of (23) is obtained from exact $L(z)$ in using method of the article [15]. The approximation in (23) is the same used in previous calculation.

In summary we have the global MSE $\sigma_{\epsilon}^{2}$ :

$$
\sigma_{\epsilon}^{2} \simeq \sigma_{w}^{2} \frac{3 K_{1}}{4}+\sigma_{\alpha}^{2} \frac{6 \pi^{4}\left(f_{d} T\right)^{4}}{K_{1}^{4}}
$$

We use the expression of (18) in (24), then we search $\sigma_{u}^{2}$ that minimizes $\sigma_{\epsilon}^{2}$. We obtain:

$$
\sigma_{u}^{2}(\mathrm{MAV})=4 \pi^{\frac{16}{5}}\left(\sigma_{\alpha}^{2}\left(f_{d} T\right)^{4} \sqrt{\sigma_{w}}\right)^{\frac{4}{5}}
$$

According to (5), $\sigma_{u}^{2}$ can be expressed in terms of $a_{1}, a_{2}$ :

$$
\sigma_{u}^{2} \simeq 2 \sigma_{\alpha}^{2}\left(1+a_{2}\right)\left(1-a_{1}-a_{2}\right)
$$

assuming that $1-a_{2} \simeq 2,1+a_{1}-a_{2} \simeq 4,1+a_{2} \ll 1$ and $1-a_{1}-a_{2} \ll 1$.

From equations (25) and (26) we deduce the following relation between $a_{1}$ and $a_{2}$ when the MAV criterion is approximately satisfied :

$$
a_{1}=-\frac{a_{2}^{2}+2 \pi^{\frac{16}{5}}\left(\left(f_{d} T\right)^{4} \sqrt{\sigma_{w}}\right)^{\frac{4}{5}}\left(\sigma_{\alpha}^{2}\right)^{-\frac{1}{5}}-1}{a_{2}+1}
$$

Equation (27) makes it possible to set $a_{1}$ as a function of $a_{2}$ and thus simplifies the search for one parameter instead of two : the asymptotic MSE is measured by "Monte-Carlo" simulation for a grid of values of $a_{2}$, and the value of $a_{2}$ corresponding to the minimum MSE is recorded. Figure 2 shows the experimental value of optimal $a_{2}$ as a function of $f_{d} T$ for different SNR. The curves show an approximative linear behavior of type $a_{2}=-1+\gamma f_{d} T$, that depends on the SNR. Figure 3 shows that $\gamma=\frac{2}{3}$ (optimal for SNR $=20 \mathrm{~dB}$ ) is a good compromise. Therefore, we impose :

$$
a_{2}=-1+\frac{2}{3} f_{d} T
$$

Table (I) gives the values of $\mathrm{AR}(2)$ parameters for various $f_{d} T$ and $\mathrm{SNR}=20 \mathrm{~dB}$.

\begin{tabular}{|l|c|c|r|}
\hline$f_{d} T$ & $10^{-4}$ & $10^{-3}$ & $10^{-2}$ \\
\hline \hline$a_{1}$ & 1.99993325954 & 1.99932163831 & 1.9914979809 \\
\hline$a_{2}$ & -0.999934 & -0.99934 & -0.9934 \\
\hline$r$ & 0.99996666611 & 0.999666611 & 0.996661092 \\
\hline$f_{\mathrm{AR}(2)} T / f_{d} T$ & 0.429 & 0.541 & 0.684 \\
\hline
\end{tabular}

TABLE I: Example of values given by (8), (27) and (28) for different values of $f_{d} T$ for $\mathrm{SNR}=20 \mathrm{~dB}$.

And the corresponding optimal MSE obtained by injecting (18) and (25) in (24) results in :

$$
\sigma_{\epsilon}^{2}(\mathrm{AR}(2)-\mathrm{MAV})=\frac{15}{8} \pi^{\frac{4}{5}}\left(\sigma_{\alpha}^{2}\right)^{\frac{1}{5}}\left(f_{d} T \sigma_{w}^{2}\right)^{\frac{4}{5}}
$$

These MSE expression is close to the one obtained by using (non-stationary) second-order random walk model instead of the stationary $\mathrm{AR}(2)$ model $[5]\left(\frac{\sigma_{\epsilon}^{2}(\mathrm{RW}(2)-\mathrm{MAV})}{\sigma_{\epsilon}^{2}(\mathrm{AR}(2)-\mathrm{MAV})}=2^{\frac{2}{5}}\right)$.

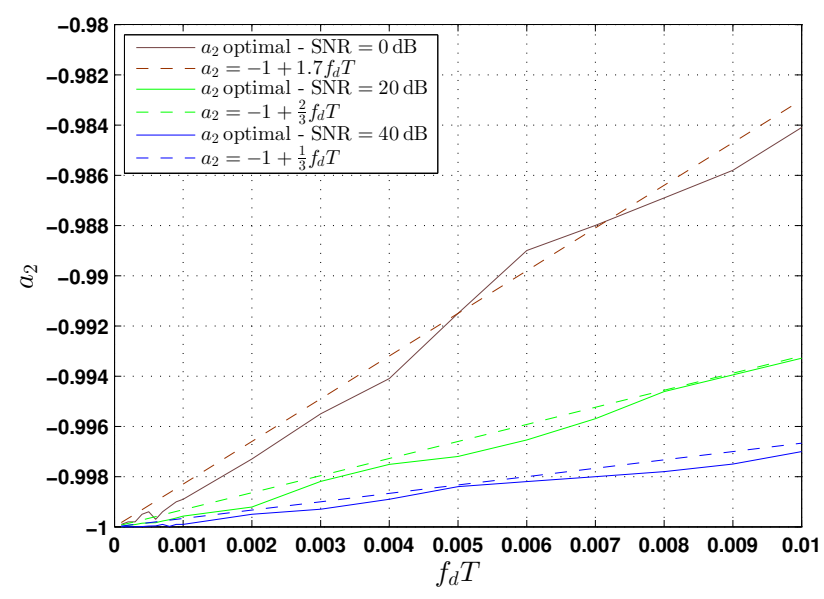

Fig. 2: Experimental values and linear approximation of optimal $a_{2}$ in terms of $f_{d} T$ for $\mathrm{SNR}=0,20$ and $40 \mathrm{~dB}$.

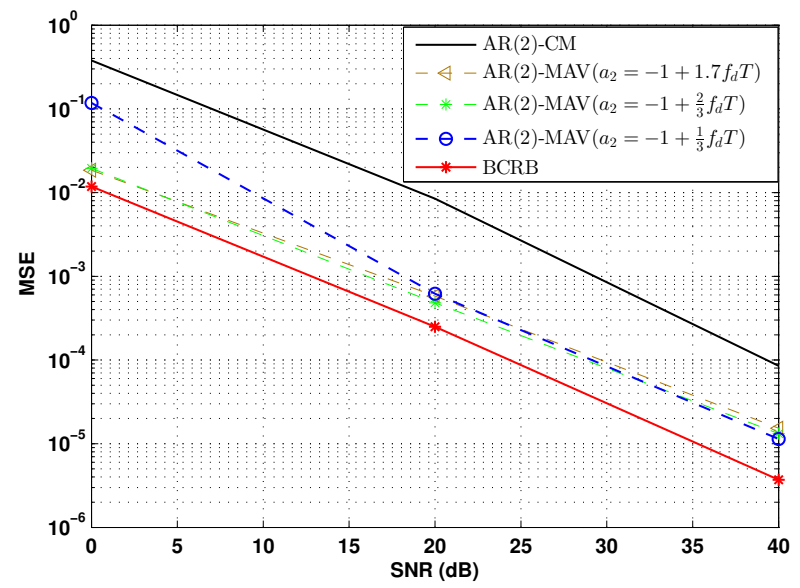

Fig. 3: Variations of MSE in terms of SNR for different imposed constraints $\left(a_{2}=-1+\gamma f_{d} T\right)$ for $f_{d} T=10^{-3}$.

\section{Simulation, DisCUSSION AND COMPARISON TO OTHER MODELS}

In [3], [4] it was shown that for low Doppler frequency and low SNR, the AR(1)-MAV coefficient $a$ noted $a_{M A V}$ allows to minimize the asymptotic estimation error variance $\sigma_{\epsilon}^{2}$ of the $\mathrm{AR}(1)-\mathrm{MAV}$ is:

$$
a_{M A V}=\sqrt{1-4\left(\left(\pi f_{d} T\right)^{4} \frac{\sigma_{w}^{2}}{\sigma_{\alpha}^{2}}\right)^{\frac{1}{3}}}
$$

And the closed-form expression of the corresponding asymptotic estimation error variance is :

$$
\sigma_{\epsilon}^{2}(\mathrm{AR}(1)-\mathrm{MAV}) \simeq \frac{3}{2} \cdot \pi^{\frac{2}{3}}\left(\sigma_{\alpha}^{2}\right)^{\frac{1}{3}}\left(f_{d} T \sigma_{w}^{2}\right)^{\frac{2}{3}}
$$

Comparing equations (29) and (31) we have :

$$
\sigma_{\epsilon}^{2}(\mathrm{AR}(2)-\mathrm{MAV})<\sigma_{\epsilon}^{2}(\mathrm{AR}(1)-\mathrm{MAV})
$$




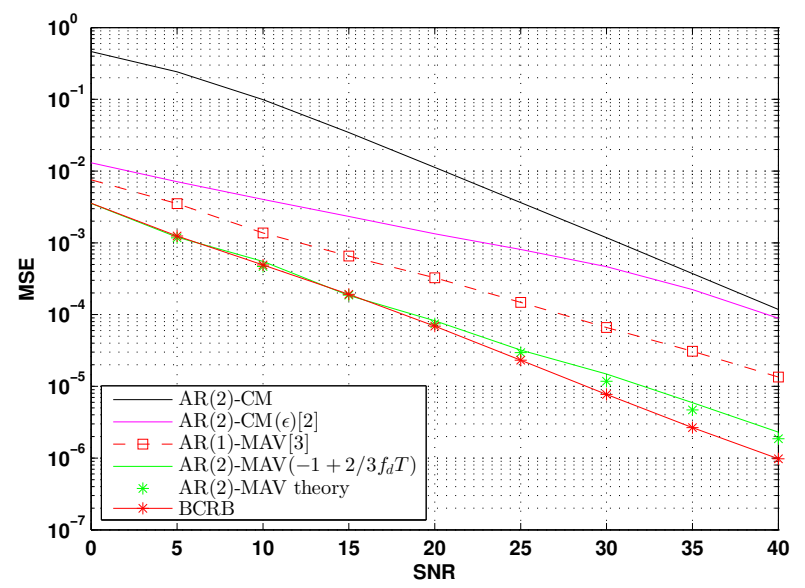

(a) $f_{d} T=10^{-4}$

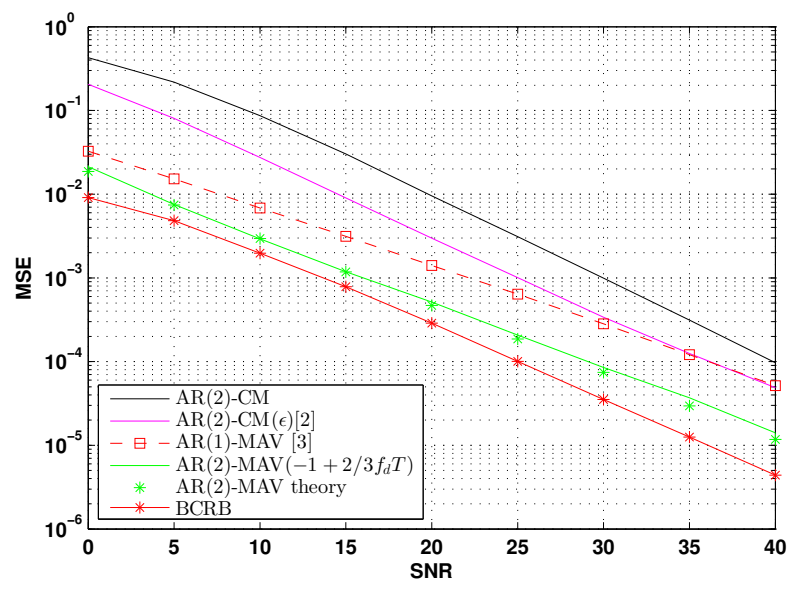

(b) $f_{d} T=10^{-3}$

Fig. 4: MSE of the Kalman filters for proposed AR(2)-MAV in terms of SNR for $f_{d} T=10^{-4}$ and $f_{d} T=10^{-3}$.

Equation (32) is valid for usual range of SNR.

Figure 4 compares the asymptotic MSE of the KF obtained by Monte-carlo simulations with AR(1)-MAV [3], AR(2)-CM with $\epsilon$ [2], $\mathrm{AR}(2)-\mathrm{CM}$ and proposed (approximate) $\mathrm{AR}(2)$ MAV. We also plot the on-line BCRB [16] and equation (29) as references. We verify by these figures that :

- For the AR(2)-MAV, the MSE computed by simulation is very close to $\sigma_{\epsilon}^{2}(\mathrm{AR}(2)-\mathrm{MAV})$ obtained by the closedform expression in (29) thus validating the theoretical analysis.

- An important point is that with the proposed $\mathrm{AR}(2)$ MAV and its optimization, we are closer to the BCRB than with AR(1)-MAV for normalized Doppler frequencies $f_{d} T \leq 10^{-3}$ which validates our theoretical analysis. This point confirms the advantage of using an approriate second order modeling versus a first order in slow fading [5].

- Comparing with the $\mathrm{AR}(2)-\mathrm{CM}$ and to the improved version [2] ( $\mathrm{AR}(2)-\mathrm{CM}(\epsilon))$ where parameters are generated by adding $\epsilon$ to the diagonal of the autocorrelation matrix, we found that the MSE of these models are far from the BCRB and higher than proposed (approximate) AR(2)-MAV.

\section{CONClusion}

Our study deals with the estimation of flat fading Rayleigh channels with Jakes spectrum using a Kalman filter (KF) with a second order autoregressive model $(\operatorname{AR}(2))$. We first show from simulation that it can be pertinent to impose a linear relation between $a_{2}$ of the $\operatorname{AR}(2)$ parameters and the normalized Doppler-frequency $f_{d} T$. Once imposing this relation, we then derive closed-form expression for the tuning of the other parameter $a_{1}$ of the AR(2) model. We then get a suboptimal adjustement of the parameters that approximately satisfies the MAV criterion (minimization of the asymptotic error variance). Simulation results show better performance in terms of MSE compared to the literature.

This work was supported by the ELSAT2020 project, cofinanced by the European Union with the European Regional Development Fund, the French state and the Hauts of France Region Council.

\section{REFERENCES}

[1] R. H. Clarke, "A statistical theory of mobile radio reception," Bell Labs Technical Journal, (1968).

[2] K. E. Baddour and N. C. Beaulieu, "Autoregressive models for fading channel simulation," in Global Telecommunications Conference, 2001. GLOBECOM '01. IEEE, vol. 2, 2001.

[3] S. Ghandour-Haidar, L. Ros, and J.-M. Brossier, "On the use of firstorder autoregressive modeling for Rayleigh flat fading channel estimation with Kalman filter," Signal Processing, vol. 92, 2012.

[4] S. Ghandour-Haidar, "Estimation de canal à évanouissements plats dans les transmissions sans fils à relais multibonds," Ph.D. dissertation, Université de Grenoble, 2014.

[5] L. Ros and E. P. Simon, "Second-order modeling for Rayleigh flat fading channel estimation with Kalman Filter," in 2011 17th International Conference on Digital Signal Processing (DSP), July 2011, pp. 1-6.

[6] H. Shu, L. Ros, and E. P. Simon, "Simplified Random-Walk-ModelBased Kalman Filter for Slow to Moderate Fading Channel Estimation in OFDM Systems," IEEE Trans. Signal Process., vol. 62, Aug 2014.

[7] H. Shu, E. P. Simon, and L. Ros, "Third-Order Kalman Filter: Tuning and Steady-State Performance," IEEE Signal Process Lett., vol. 20, 2013.

[8] G. Eshel, "The yule walker equations for the AR coefficients ," Internet resource, vol. 2, p. 68-73., 2003.

[9] L. Lindbom, "Simplified Kalman estimation of fading mobile radio channels: high performance at LMS computational load," in IEEE International Conference on Acoust. Speech Signal Process., vol. 3, April 1993, pp. 352-355 vol.3.

[10] H.-Y. Wu and A. Duel-Hallen, "On the performance of coherent and noncoherent multiuser detectors for mobile radio CDMA channels," in ICUPC, vol. 1, Sep 1996, pp. 76-80 vol.1.

[11] W. W.-S. Wei, Time series analysis. Addison-Wesley publ, 1994.

[12] A. Fathi and N. Sharifan, "A classic new method to solve quartic equations," Applied and Computational Mathematics, vol. 2, no. 2, pp. 24-27, 2013.

[13] D. Vaughan, "A nonrecursive algebraic solution for the discrete Riccati equation," IEEE Trans. Autom. Control, vol. 15, no. 5, Oct 1970.

[14] B. Ekstrand, "Analytical Steady State Solution for a Kalman Tracking Filter," IEEE Trans. Aerosp. Electron. Syst., vol. AES-19, no. 6, 1983.

[15] R.Winkelstein, "Closed form evaluation of symmetric Two-sided Complex integrals," communications Systems Research Section, July and August 1981.

[16] H. Hijazi and L. Ros, "Bayesian Cramer-Rao Bounds for Complex Gain Parameters Estimation of Slowly Varying Rayleigh Channel in OFDM Systems," Signal Processing, vol. 89, no. 1, Jan. 2009. 